\title{
The development and evaluation of a multi-epitope antigen as a serodiagnostic marker of Toxoplasma gondii infection
}

\author{
Abbas Alibakhshi, ${ }^{\mathrm{A}, \mathrm{B}, \mathrm{D}}$, Mojgan Bandehpour ${ }^{\mathrm{C}, \mathrm{F}}$, Zarin Sharifnia ${ }^{\mathrm{C}, \mathrm{E}}$, Bahram Kazemi ${ }^{\mathrm{A}, \mathrm{F}}$ \\ Cellular and Molecular Biology Research Center, Shahid Beheshti University of Medical Sciences, Tehran, Iran \\ A - research concept and design; $B$ - collection and/or assembly of data; $C$ - data analysis and interpretation; \\ $D$ - writing the article; $E$ - critical revision of the article; $F$ - final approval of the article
}

Address for correspondence

Mojgan Bandehpour

E-mail: bandehpour@gmail.com

Funding sources

None declared

Conflict of interest

The present study was conducted in the Cellular \& Molecular Biology Research Center of Shahid Beheshti University of Medical Sciences, Tehran, Iran. This article was extracted from Abbas Alibakhshi's Ph.D. thesis. The project was funded by the Deputy of Shahid Beheshti University of Medical Sciences (grant No. 4971) and the experiments were carried out in concordance with ethics code SBMU.REC.1393.749.

Received on July 17, 2017

Reviewed on March 1, 2018

Accepted on February 18, 2019

Published online on June 23, 2020

\begin{abstract}
Background. Toxoplasma gondii (T. gondii) is a ubiquitous protozoan parasite which causes a serious disease called toxoplasmosis. The high prevalence of T. gondii infection has attracted a great deal of interest in its diagnosis and treatment. The use of pure antigens shows high sensitivity and specificity, but challenges such as cross-reactivity remain diagnostic difficulties.
\end{abstract}

Objectives. The aim of this study was to use 3 surface antigens (SAGs) of T. gondii to design gene-encoding a multi-epitope and immunogenic protein as a serodiagnostic marker.

Material and methods. The multi-epitope antigen was expressed using Escherichia coli BL21 (DE3) cells and purified using affinity chromatography. To evaluate acute toxoplasmosis, 95 human sera with anti-T. gondii IgG, 25 human sera without anti-T. gondii Ig G and 6 serum samples with nosocomial infections were collected and submitted to an enzyme-linked immunosorbent assay (ELISA) analysis. The potential of purified protein as a diagnostic marker of T. gondii infection was also investigated using ELISA analysis.

Results. The western blot analysis for both protein expression and purification confirmed that the protein was expressed and purified successfully. The results of validation showed a sensitivity of $72.6 \%$ and a specificity of $90.3 \%$ for recombinant ELISA.

Conclusions. Although this protein showed potential for detecting T. gondii, the sensitivity and specificity were lower than in tests that use the whole body of the parasite.

Key words: diagnosis, Toxoplasma gondii, multi-epitope antigen, surface antigens
Cite as

Alibakhshi A, Bandehpour M, Sharifnia Z, Kazemi B.

The development and evaluation of a multi-epitope antigen as a serodiagnostic marker of Toxoplasma gondii infection. Adv Clin Exp Med. 2020;29(6):669-675.

doi:10.17219/acem/104554

DOI

10.17219/acem/104554

Copyright

Copyright by Author(s)

This is an article distributed under the terms of the

Creative Commons Attribution 3.0 Unported (CC BY 3.0)

(https://creativecommons.org/licenses/by/3.0/) 


\section{Introduction}

Toxoplasma gondii, a ubiquitous protozoan parasite, is one of the most successful pathogens that can infect almost all warm-blooded animals, including humans. The primary infection is mostly asymptomatic in humans, but it can cause severe damage, especially in pregnant women and immunocompromised hosts with T-cell defects. For example, transmission from an infected mother to a fetus may lead to spontaneous abortion or stillbirth. ${ }^{1}$ Recently, it has been found that $T$. gondii infection can correlate with neuropsychiatric disorders, including depression and suicidal behavior. ${ }^{2}$

Toxoplasma gondii infection in humans occurs in 3 stages, including tachyzoite, bradyzoite and oocyst environmental stages. People can be infected through ingestion of oocytes. Tissue cysts containing bradyzoites appear in all warm-blooded animals, including humans. Rapidly replicating tachyzoites, which are responsible for acute toxoplasmosis, divide inside specialized vacuoles of the host cells. ${ }^{3}$ The complicated virulence is dependent on factors involved in the interaction between the parasite and the host in cell invasion and intracellular development, as well as factors involved in the host immune response. Resident surface proteins are among the virulence factors affecting the gliding, attachment and invasion of the parasite. In initial studies on T. gondii, P30 (surface antigen SAG1), which causes strong antibody responses, was identified as a glycosylphosphatidylinositol (GPI)-anchored surface protein. ${ }^{4}$ Later investigations led to the detection of more surface antigens in T. gondii. These antigens have different roles in pathogenesis, including attachment and invasion of the parasite to the host cells and immune modulation. Surface antigens 2, 3, 4, 5, and SAG1related sequences (SRS) are other proteins on the surface of $T$. gondii tachyzoites and bradyzoites. ${ }^{5}$

Molecular factors involved in infection diseases are generally used as serodiagnostic markers. At the same time, serological tests that measure antibodies raised against infection are the primary methods for diagnosing T. gondii infection. These tests usually measure immunoglobulin $\mathrm{M}$ (IgM, as an indication of recent infection) or immunoglobulin G (IgG) (as a demonstration of the immune status against a particular pathogen) antibody titers using laboratory methods. A panel of serological tests has been used to demonstrate specific antibodies against T. gondii. ${ }^{6} \mathrm{Al}-$ though conventional antibody screening against $T$. gondii (and also many other pathogens) is an optimal strategy to prevent and decrease mainly congenital type toxoplasmosis, the low accuracy of the crude native antigens makes them unsuitable for use in diagnostic methods. Furthermore, there are difficulties in standardizing diagnostic kits that employ native antigens. Therefore, assuming it may be fruitful to replace these antigens with alternative proteins and peptides, investigations have been inclined significantly towards alternative and standard methods with the help of suitable reagents with high diagnostic sensitivity. Recently, peptide-based antigens and poly-epitope antigens have been considered by some researchers as increasing the sensitivity and specificity of diagnostic tests for some pathogens. ${ }^{7}$ In this method, only a highly immunogenic region of an antigen in the form of a peptide or a poly-epitope protein is employed to diagnose the presence or absence of specific antibodies in human serum.

In the present study, we constructed a synthetic gene encoding epitopes and highly immunogenic regions of 3 surface genes of T. gondii, namely SAG1 (P30), SAG2 (P22) and SAG3 (P43), for the production of a poly-epitope protein as a novel reagent to detect infection caused by $T$. gondii.

\section{Material and methods}

\section{Sequence retrieval, in silico analysis and design of a poly-epitope protein}

Nucleotide sequences for 3 surface antigens (SAG1, SAG2 and SAG3) of the extremely virulent RH strain of T. gondii were obtained from the National Center for Biotechnology Information (NCBI; Bethesda, USA) Nucleotide Database. After translation into amino acid sequences, all 3 proteins were aligned with proteins from different virulent strains using the Clustal algorithm. The secondary and tertiary structures of the proteins were considered for further evaluation. Amino acid distribution was analyzed using the LRRfinder server (http://www.lrrfinder.com), and the transmembrane topology of the proteins was studied with methods available on the TMHMM server (http:// www.cbs.dtu.dk/services/TMHMM-2.0/) for investigating the location of immunogenic regions. The IEDB ${ }^{8}$ ABCpred, ${ }^{9}$ Bcepred, ${ }^{10}$ and Bepipred ${ }^{11}$ web servers were used to predict B-cell epitopes; the epitopes were selected mainly on the basis of the Kolaskar and Tongaonkar antigenicity method. ${ }^{12}$ Finally, 3 immunogenic regions were chosen from the surface antigens and then connected to each other with linker peptides. A 405-nucleotide sequence containing epitopes was synthesized into the multiple cloning sites (MCS) of the pQE30 expression vector.

\section{Expression and purification of the poly-epitope protein}

The recombinant $\mathrm{PQE30}$ vector was analyzed using polymerase chain reaction (PCR) for confirmation of the synthesized sequence. After the transformation of the recombinant vector into Escherichia coli BL21 (DE3) cells, protein expression was induced by placing $1 \mathrm{mM}$ of the final isopropyl-D-thiogalactopyranoside (IPTG) concentration in a Luria broth (LB) medium. Aliquots of the medium were removed $2 \mathrm{~h}$ and $3 \mathrm{~h}$ after the induction of protein expression. The cells were lysed in lysis 
buffer (50 mM Tris- $\mathrm{HCl}, 10 \%$ glycerol, 0.1\% Triton X-100, and $1 \mathrm{mM}$ phenylmethylsulfonyl fluoride (PMSF)). To verify and assess the protein expression, western blot analysis was carried out on protein extracts. First, 2X SDS-loading buffer (100 mM Tris-HCl pH 6.8, 20\% glycerol, 4\% SDS, $0.005 \%$ bromophenol blue, and $200 \mathrm{mM} \mathrm{DTT}$ ) was added to the proteins, and then the samples were heated at $85^{\circ} \mathrm{C}$ for $5 \mathrm{~min}$. The proteins of the cell extracts were separated on a $12 \%$ polyacrylamide gel and run in $1 \mathrm{X}$ TrisGlycine SDS buffer (TGS). The proteins were transferred to a nitrocellulose membrane sheet and blocked with $3 \%$ skimmed milk powder in phosphate buffer saline (PBS) for $1 \mathrm{~h}$. The membrane layer was washed 3 times in PBS with $0.1 \%$ Tween (PBST). Anti-polyhistidine-alkaline phosphatase (ALP) antibody in a 1:10,000 dilution was then used to incubate the membrane layer with $40 \mathrm{rpm}$ shaking for $2 \mathrm{~h}$. The washing step was repeated and finally bromo-color-indolyl-phosphate (BCIP) and nitro blue tetrazolium (NBT) were applied to detect the target protein on the membrane sheet.

After confirmation of protein expression, the aliquots taken $3 \mathrm{~h}$ after induction were used for the protein purification process. Using the pQE30 vector, a His6 tag was conjugated to the target protein. In this process, the lysis of the cells was carried out in equilibrium buffer ( $2 \mathrm{M}$ urea, $20 \mathrm{mM}$ Tris- $\mathrm{HCl}, 500 \mathrm{NaCl}, 50 \mathrm{mM}$ imidazole, $0.5 \%$ Triton $\mathrm{X}-100$, and PMSF, pH 8) and the samples were sonicated on ice for $1 \mathrm{~h}$. The lysate was centrifuged at 5,000 rpm for $20 \mathrm{~min}$, and the supernatant was poured into an $\mathrm{Ni}$ (II)based immobilized metal ion affinity chromatography column. The column was shaken for $1 \mathrm{~h}$ and then washed 3 times with washing buffer $(0.4 \mathrm{M}$ urea, $20 \mathrm{mM}$ Tris- $\mathrm{HCl}$, $500 \mathrm{NaCl}, 50 \mathrm{mM}$ imidazole, $0.5 \%$ Triton X-100, and PMSF, $\mathrm{pH} 8$ ) and finally eluted with elution buffer (0.4 M urea, $20 \mathrm{mM}$ Tris- $\mathrm{HCl}, 500 \mathrm{NaCl}$, and $500 \mathrm{mM}$ imidazole, $\mathrm{pH}$ 8). The purification process was confirmed with western blot analysis and dot-blot analysis. The western blot was carried out as described above for the confirmation of protein expression, but instead of using the anti-polyhistidine ALP antibody, we used the anti-polyhistidine antibody conjugated to horseradish peroxidase (HRP) and its chromogenic substrate, 3,3'-diaminobenzidine (DAB), along with hydrogen peroxide $\left(\mathrm{H}_{2} \mathrm{O}_{2}\right)$. For dot-blot, a drop of liquid from the purification process and a drop of flow-through were placed directly on a nitrocellulose membrane sheet; the membrane was then blocked with skimmed milk, washed, incubated with anti-polyhistidine HRP antibody and detected with $\mathrm{DAB}$, as in the process described for western blot analysis.

\section{Serum samples and indirect IgG ELISA}

The potential of purified protein as a diagnostic marker of $T$. gondii infection was investigated using enzyme-linked immunosorbent assay (ELISA) analysis. To evaluate acute toxoplasmosis, 95 human sera with anti-T. gondii IgG and
25 human sera without anti-T. gondii IgG were collected from Nilou Medical Laboratory in Tehran, Iran, as well as 6 serum samples with nosocomial infections (infections other than toxoplasmosis). All of the serum samples were first analyzed using a commercial IgG ELISA kit (Euroimmun AG, Lübeck, Germany). To set up the assay, different concentrations of purified protein in $1 \times$ PBS were prepared and used to coat a polystyrene 96-well microtiter plate, which was incubated overnight at room temperature. Afterward, the plate was washed 3 times with PBST $(1 \times$ PBS and $0.05 \%$ Tween 20$)$ and then blocked with $300 \mu \mathrm{L}$ of $1 \%$ bovine serum albumin (BSA) in $1 \times$ PBS for $1.5 \mathrm{~h}$. The plate was washed 3 times and $100 \mu \mathrm{L}$ of a series of 2 anti-T. gondii IgG-positive and 2 anti-T. gondii IgGnegative serum samples that had already been prepared using $1 \times$ PBS were added to each well. The plate was left at room temperature for $2.5 \mathrm{~h}$. The plate was again washed 3 times; $100 \mu \mathrm{L}$ of HRP-conjugated rabbit anti-human IgG (1:10,000 dilution in $1 \times$ PBS) was added to each well; and the plate was left at room temperature for $1 \mathrm{~h}$. After rewashing the plate, $100 \mu \mathrm{L}$ of 3,3',5,5'-tetramethylbenzidine (TMB) substrate was added, and after $15 \mathrm{~min}$ at room temperature, the reaction was stopped with $50 \mu \mathrm{L}$ of $2 \mathrm{~N}$ $\mathrm{H}_{2} \mathrm{SO}_{4}$. The optical density (OD) of each well was measured with an ELISA reader at wavelength $450 \mathrm{~nm}$ with a reference wavelength of $630 \mathrm{~nm}$. The assay was repeated for all of the serum samples at a 1:200 dilution and with $1 \mu \mathrm{g} / \mathrm{mL}$ of purified protein. The mean \pm standard deviation (SD) of all 25 anti-Toxoplasma IgG-negative serum samples was considered the cut-off value.

\section{Statistical analysis}

The sensitivity and specificity were calculated using the VassarStats website (http://vassarstats.net/) with the following formulae: sensitivity $=\mathrm{TP} /(\mathrm{TP}+\mathrm{FN}) \times 100$ and specificity $=\mathrm{TN} /(\mathrm{TN}+\mathrm{FP}) \times 100(\mathrm{TP}-$ true positive, FN - false negative, TN - true negative, FP - false positive). The sensitivity and specificity were calculated with receiver operating characteristic (ROC) curve using Prism graphing and statistics software (GraphPad Software, San Diego, USA).

\section{Results}

\section{Design of the poly-epitope protein}

The alignment of amino acid sequences of 3 surface antigens obtained from the RH strain showed high similarity with other pathogenic strains of $T$. gondii. The selection of immunological regions was based on the distribution of amino acids and transmembrane topology. The analysis of the predictions obtained from the TMHMM server showed that all 3 proteins were embedded on the cell surface and therefore available to the immune system 

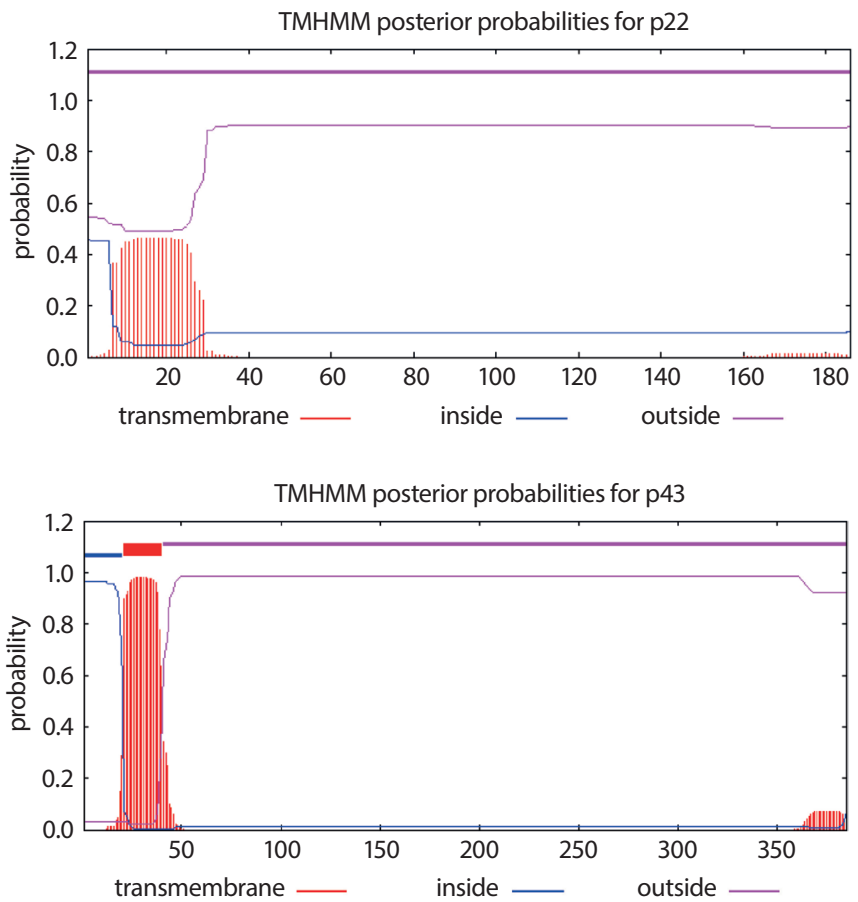

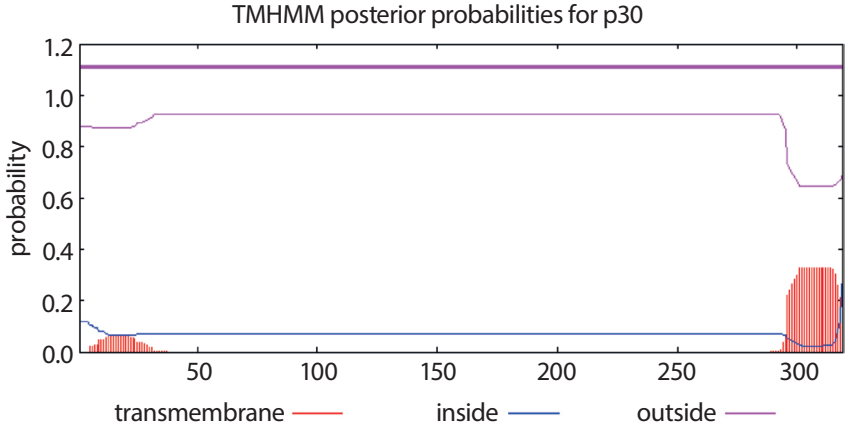

Fig. 1. Prediction of transmembrane topology of the p22, p30 and p43 proteins

(Fig. 1). The epitopes with high scores were selected, and the region with the most epitopes from each protein was identified. For SAG1, the amino acids 198-226 were selected, for SAG2 the amino acids 116-164 were selected and for SAG3 the amino acids 234-276 were selected; subsequently, a poly-epitope protein was designed (Fig. 2).

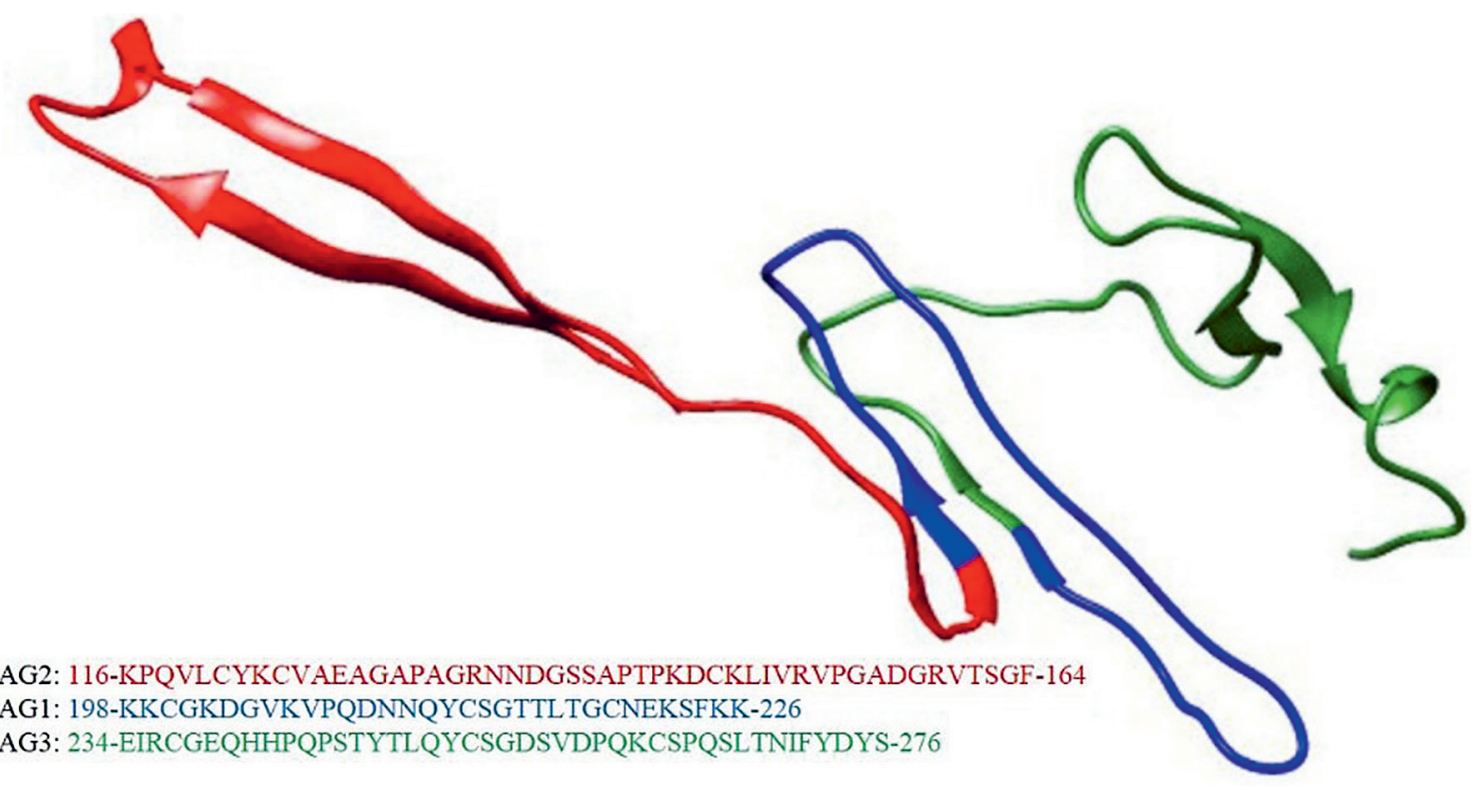

Fig. 2. Predicted tertiary structure of the multiepitope protein by the Phyre2 protein fold recognition server

SAG1: 198-KKCGKDGVKVPQDNNQYCSGTTLTGCNEKSFKK-226

SAG3: 234-EIRCGEQHHPQPSTYTLQYCSGDSVDPQKCSPQSLTNIFYDYS-276
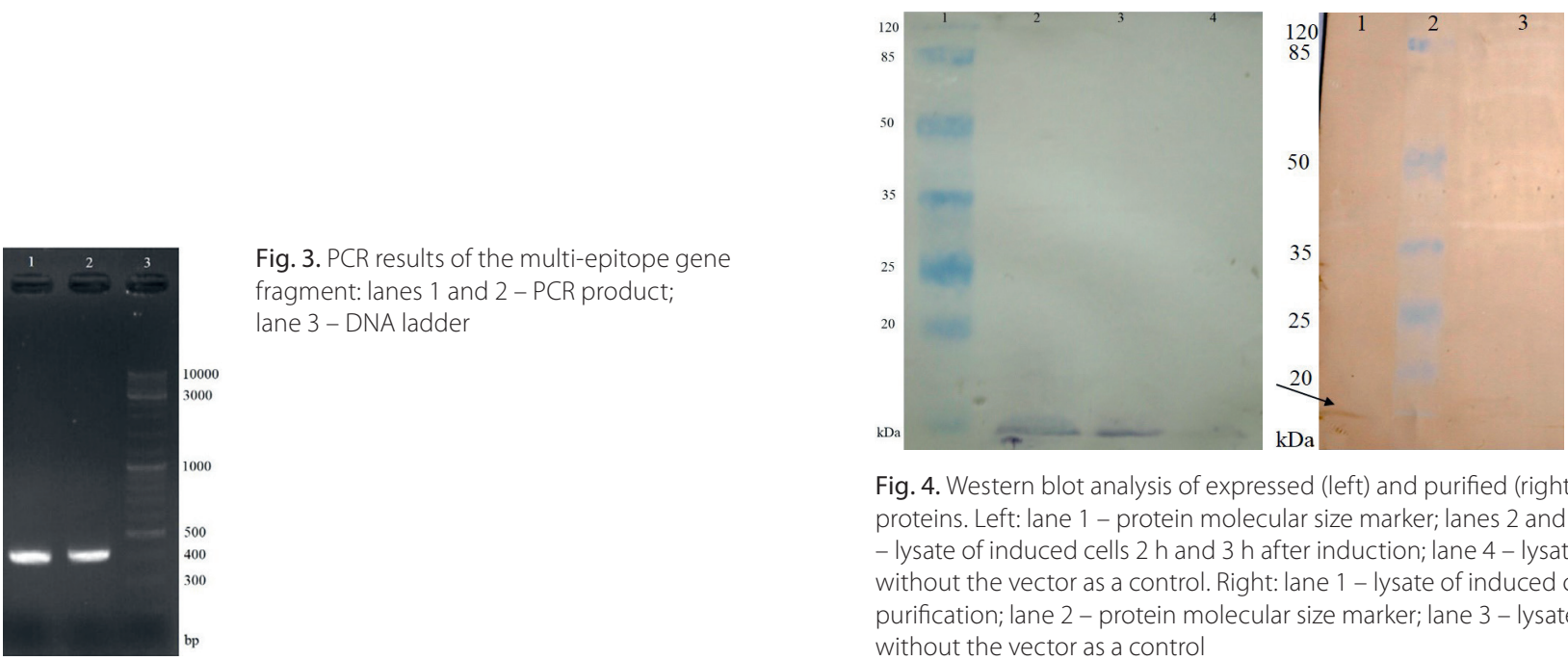

Fig. 3. PCR results of the multi-epitope gene fragment: lanes 1 and $2-$ PCR product; lane 3 - DNA ladder

Fig. 4. Western blot analysis of expressed (left) and purified (right) proteins. Left: lane 1 - protein molecular size marker; lanes 2 and 3

- lysate of induced cells $2 \mathrm{~h}$ and $3 \mathrm{~h}$ after induction; lane 4 - lysate of cells without the vector as a control. Right: lane 1 - lysate of induced cells afte purification; lane 2 - protein molecular size marker; lane 3 - lysate of cells without the vector as a control 
The size of the multi-epitope gene ( $405 \mathrm{bp}$ ) was confirmed with PCR (Fig. 3). The calculation of molecular weight showed a protein with a molecular weight of about $12 \mathrm{kDa}$.

\section{Analysis of the purified protein}

The western blot analysis of protein expression revealed that protein is expressed at both $2 \mathrm{~h}$ and $3 \mathrm{~h}$ after induction. The expressed protein was purified using affinity chromatography, after which western blot and dot-blot analysis confirmed that the protein was purified successfully but not completely (Fig. 4).

\section{IgG ELISA analysis}

The cut-off value was 1.65 . From 95 serum samples with anti-T. gondii IgG, 69 cases were positive by poly-epitope protein. Two cases from the negative serum samples and 1 case from the nosocomial infections were also positive (Fig. 5). The comparison of the results of the commercial and recombinant ELISA assays indicated a sensitivity of $72.6 \%$ and a specificity of $90.3 \%$ for the recombinant ELISA (Table 1). Moreover, the result of the ROC curve analysis using GraphPad Prism software showed the area under the curve as 0.93 (0.88 to 0.97$)$ with a $95 \%$ confidence interval (95\% CI) (Fig. 6).

Table 1. Sensitivity and specificity of multi-epitope IgG ELISA for diagnosis of toxoplasmosis

\begin{tabular}{|l|c|c|c|c|}
\hline Sensitivity [\%] & Specificity [\%] & Positive predictive value [\%] & Negative predictive value [\%] & Agreement [\%] \\
\hline 72.6 & 90.3 & 95.8 & 51.8 & 76.9 \\
\hline
\end{tabular}

Agreement: $(\mathrm{TP}+\mathrm{TN}) /(\mathrm{TP}+\mathrm{TN}+\mathrm{FP}+\mathrm{FN}) \times 100$.

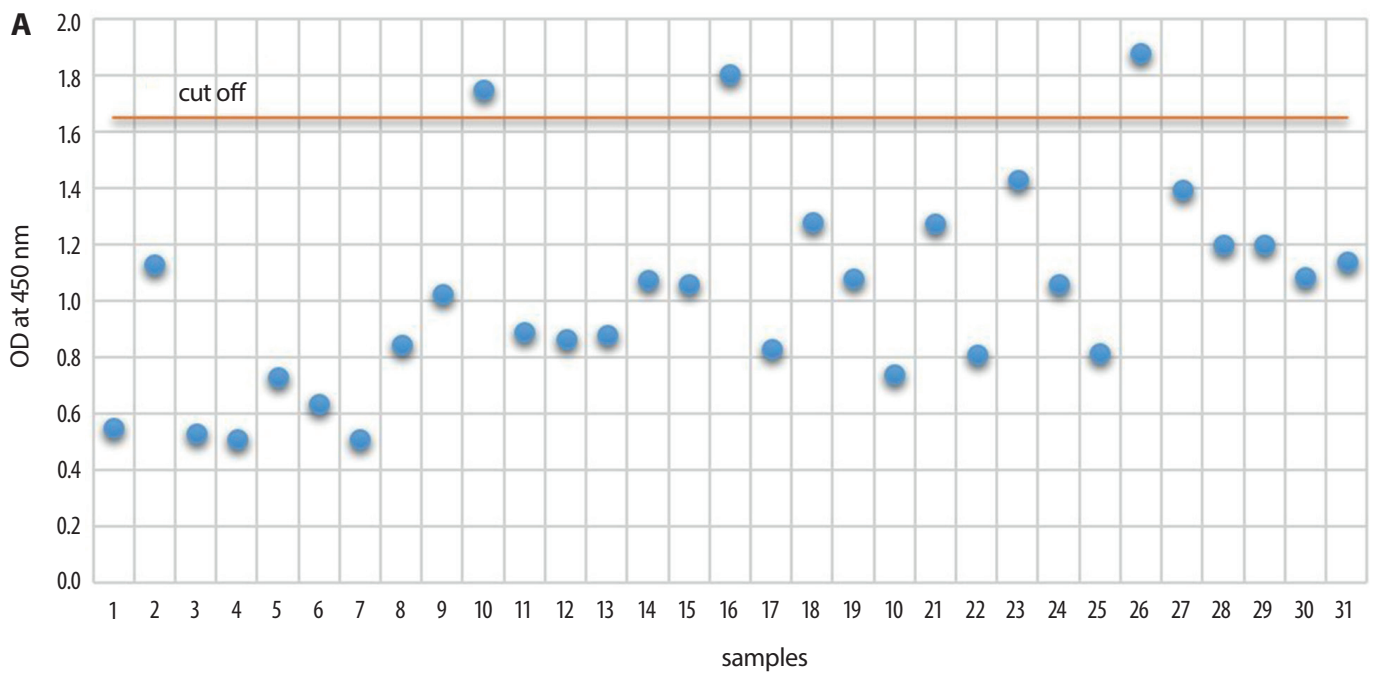

Fig. 5. Results of IgG ELISA

A - negative samples; B - positive samples.

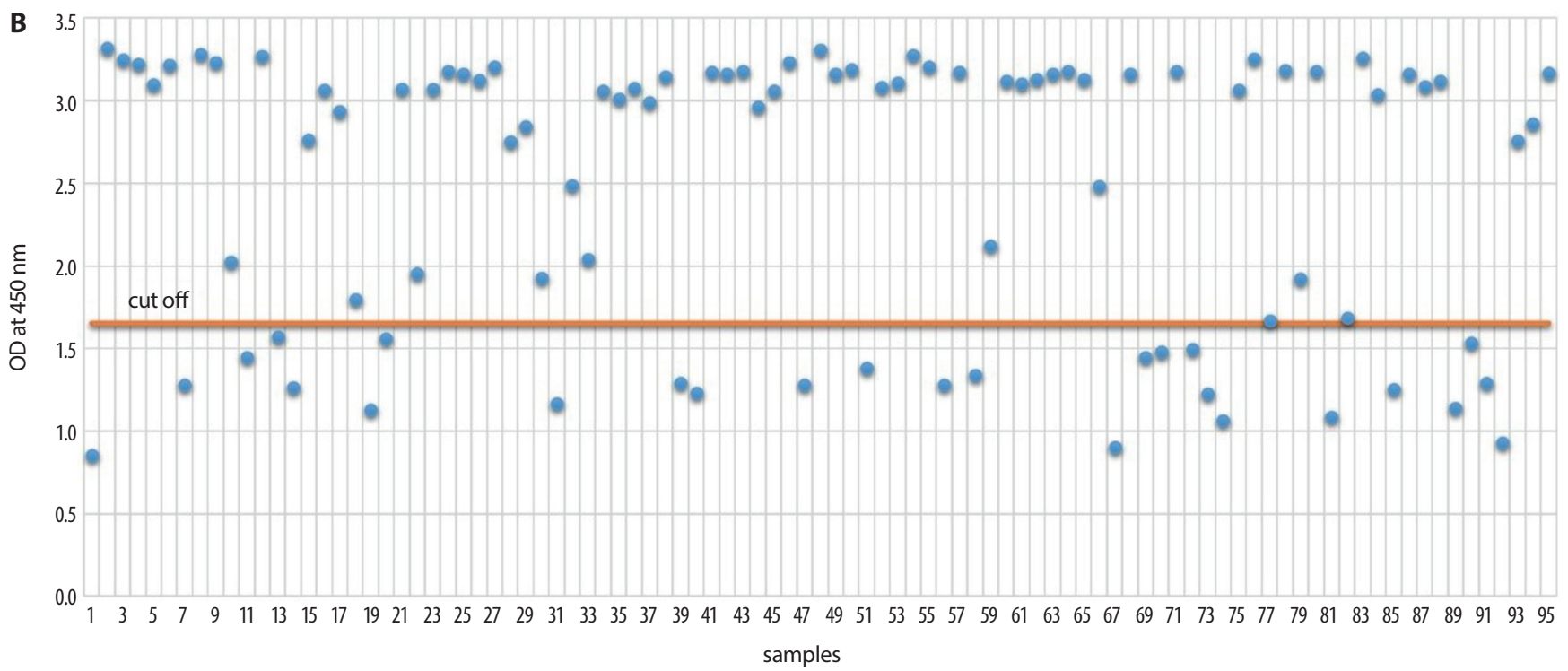



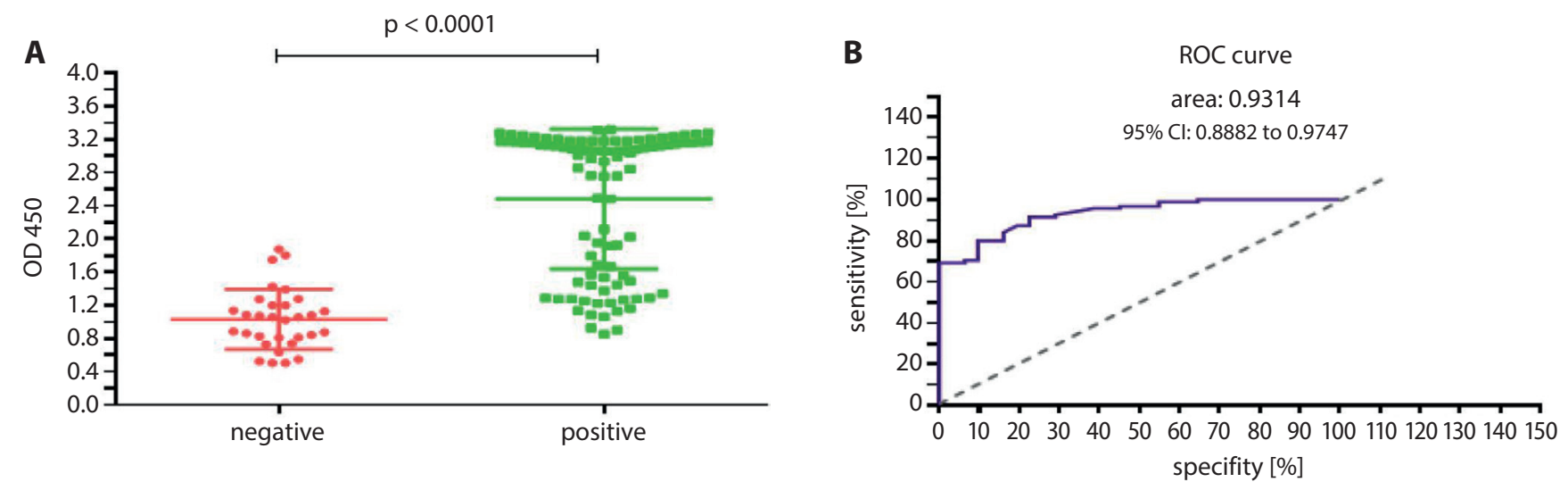

Fig. 6. Results of ROC analysis in the assessment of diagnostic marker utility. A - optical density values of 2 anti-T. gondii lgG-positive and 2 anti-T. gondii IgG-negative serum samples; B - ROC curve; graphical plot of sensitivity vs specificity

\section{Discussion}

The current study evaluated the potential of a polyepitope protein comprising 3 immunological regions obtained from surface antigens of $T$. gondii as a diagnostic tool. The selection of epitopes was based on various factors, mainly regions with high immunogenicity. For example, the nature of a given polarity of amino acids and also the number of hydrophilic residues in the protein and thus the solubility of the protein have a remarkable effect on the immunogenicity of antigens. Moreover, the cellular location of antigens and their accessibility to the immune system may be an important factor in designing diagnostic proteins. The in silico analysis exhibited that all 3 surface antigens in this study possess more hydrophilic amino acids. They also have significant elements accessible to the immune system. ${ }^{13}$ Studies have also shown that these proteins can be considered in controlling strategies against toxoplasmosis, including diagnosis and vaccine design. ${ }^{14}$ Most of the diagnostic studies have been based on whole antigens.

Native antigens have shown promising results in detecting pathogenic microorganisms, including T. gondii, but their use entails problems like high price, low sensitivity and low specificity. This may be due to different reasons, such as cross-reactivity. A new alternative method is the use of a multi-epitope-based antigen with the help of in silico prediction tools in which only highly immunogenic regions or only regions which do not show cross-reactivity with other microorganism antigens are selected. This study showed that although a multi-epitope protein comprising epitopes from SAG1, SAG2 and SAG3 can detect some of the individuals suffering from toxoplasmosis, we need further investigation to improve the diagnosis of this infection by modified epitopes, by selecting other epitopes and even by involving other antigens of $T$. gondii. The current multi-epitope protein showed that such proteins could be a potential candidate for use in diagnostic work; however, more studies as well as other methods are needed to increase the sensitivity and specificity of multi-epitope antigens to use as target tools in diagnostic kits. Hajissa et al. produced a synthetic protein consisting of epitopes obtained from 3 T. gondii antigens for use as a diagnostic marker and vaccine candidate. ${ }^{7}$ In their study, 9 epitopes from 3 antigens (SAG1, GRA2 and GRA7) were used for the construction of a multi-epitope protein. GRAs are dense granule antigens in T. gondii which cause high induction of antibody response in humans. The ability of this designed protein to detect anti-Toxoplasma antibodies was analyzed with western blot and ELISA, which have shown that the protein can detect acute $T$. gondii infection with high sensitivity and specificity.

The most important thing in designing these proteins is choosing appropriate antigens with high immunogenicity, and then selecting epitopes with the highest score using web servers with high predictive potential. Therefore, in silico analysis and software-based prediction techniques are vital steps toward a promising multi-epitope protein for use in diagnostic kits. Studies have shown that for T. gondii, surface antigens are ideal candidates for diagnosis as well as for vaccine design; each of these antigens alone has been studied as a potential diagnostic marker. Khanaliha et al. evaluated 3 surface antigens (SAGs 1, 2 and 3) separately for their diagnostic usefulness and efficacy. Recombinant SAG1, SAG2 and SAG3 produced in $E$. coli showed suitable antigens to detect antiToxoplasma IgG with 93.6\%, 95.4\% and 100\% sensitivity, and specificity of $92.9 \%, 89.4 \%$ and $91.2 \%$, respectively. ${ }^{14}$ The relatively low values in our study could indicate that better designing and further investigation in the selection of epitopes is required.

The differences between the current study and those mentioned above could be due to various reasons. Protein conformation and protein folding are influenced by the reaction conditions, such as $\mathrm{pH}$ and temperature during experiments in artificially differentiated conditions as opposed to the natural synthesis of the protein. Therefore, 
a test that uses the whole body of the parasite has greater sensitivity and specificity than the tests using selected epitopes in this study, and may achieve better results by adding other antigen-determinant epitopes.

\section{References}

1. Opsteegh M, Kortbeek TM, Havelaar AH, van der Giessen JW. Intervention strategies to reduce human Toxoplasma gondii disease burden. Clin Infect Dis. 2015;60(1):101-107.

2. Hsu PC, Groer M, Beckie T. New findings: Depression, suicide, and Toxoplasma gondii infection. J Am Assoc Nurse Pract. 2014;26(11): 629-637.

3. Blader IJ, Coleman BI, Chen CT, Gubbels MJ. Lytic cycle of Toxoplasma gondii: 15 years later. Annu Rev Microbiol. 2015;69:463-485.

4. Dubremetz JF, Lebrun M. Virulence factors of Toxoplasma gondii. Microbes Infect. 2012;14(15):1403-1410.

5. Lekutis C, Ferguson DJ, Grigg ME, Camps M, Boothroyd JC. Surface antigens of Toxoplasma gondii: Variations on a theme. Int J Parasitol. 2001;31(12):1285-1292.

6. Montoya JG. Laboratory diagnosis of Toxoplasma gondii infection and toxoplasmosis. J Infect Dis. 2002;185(Suppl 1):S73-S82.
7. Hajissa K, Zakaria R, Suppian R, Mohamed Z. Design and evaluation of a recombinant multi-epitope antigen for serodiagnosis of Toxoplasma gondii infection in humans. Parasit Vectors. 2015;8:315.

8. Vita R, Overton JA, Greenbaum JA, et al. The immune epitope database (IEDB) 3.0. Nucleic Acids Res. 2015;43(Database issue):D405-D412.

9. Saha S, Raghava GP. Prediction of continuous B-cell epitopes in an antigen using recurrent neural network. Proteins. 2006;65(1):40-48.

10. Saha S, Raghava GPS. BcePred: Prediction of continuous B-cell epitopes in antigenic sequences using physico-chemical properties. In: Nicosia G, Cutello V, Bentley PJ, Timmis J, eds. Artificial Immune Systems: Third International Conference, ICARIS 2004, Catania, Sicily, Italy, September 13-16, 2004 Proceedings. Berlin-Heidelberg; Springer 2004:197-204.

11. Larsen JE, Lund O, Nielsen M. Improved method for predicting linear B-cell epitopes. Immunome Res. 2006;2:2.

12. Kolaskar AS, Tongaonkar PC. A semi-empirical method for prediction of antigenic determinants on protein antigens. FEBS Lett. 1990;276 $(1-2): 172-174$.

13. Tomavo S, Schwarz RT, Dubremetz JF. Evidence for glycosyl-phosphatidylinositol anchoring of Toxoplasma gondii major surface antigens. Mol Cell Biol. 1989;9(10):4576-4580.

14. Khanaliha K, Motazedian MH, Kazemi B, Shahriari B, Bandehpour M, Sharifniya Z. Evaluation of recombinant SAG1, SAG2, and SAG3 antigens for serodiagnosis of toxoplasmosis. Korean J Parasitol. 2014; 52(2):137-142. 